\title{
Bilateral Adrenal Calcification caused by a previous Streptococus Mitis Septicaemia
}

\author{
M. Ahmed' ${ }^{1}$, P. Moulik², A. Macleod ${ }^{2}$
}

\section{SPR endocrinology; 2. Consultant Physician and Endocrinologist}

This 23 year old man was noted incidentally to have bilateral adrenal calcification on CT scan of his abdomen for chronic abdominal pain. He had normal growth and milestones with no neonatal events. Aged 10 years he was admitted briefly to a high dependency unit with circulatory shock and hyperthermia, with isolation of Streptococcus mitis from blood culture. One year later he was noted to have bilateral adrenal calcification on abdominal XR, and a short Synacthen $(250 \mathrm{mcg})$ test was considered normal (baseline cortisol of $303 \mathrm{nmol} / /$ rising to a peak of $543 \mathrm{nmol} / \mathrm{l})$. Current investigations reveal normal adrenal function including a Short synacthen test; which revealed normal response with normal base line cortisol of $440 \mathrm{nmol} / /$ rising to a peak of 620 $\mathrm{nmol} / \mathrm{l}$ with normal serum renin and ACTH.

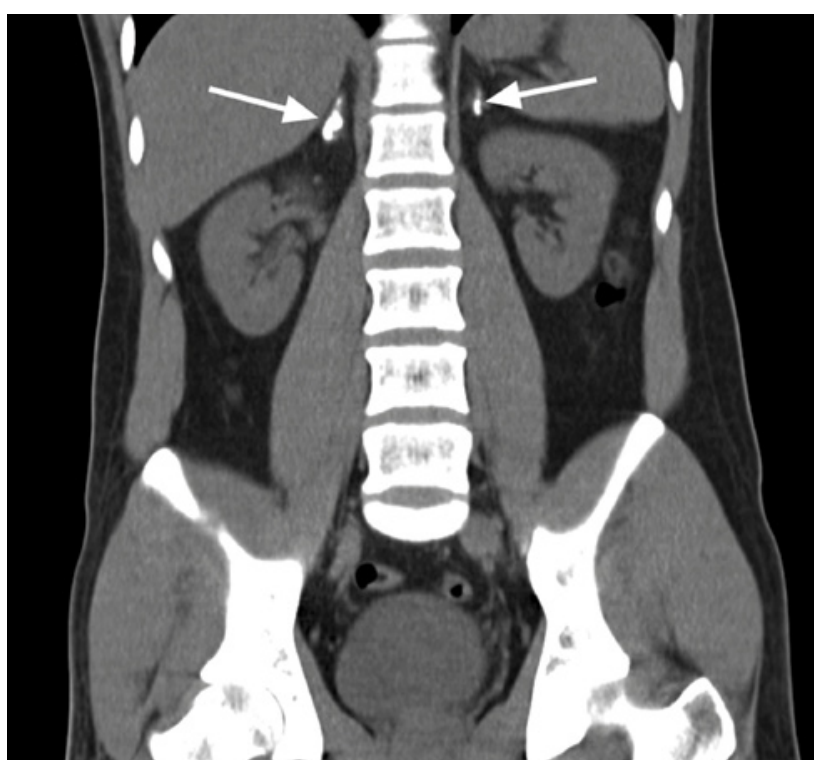

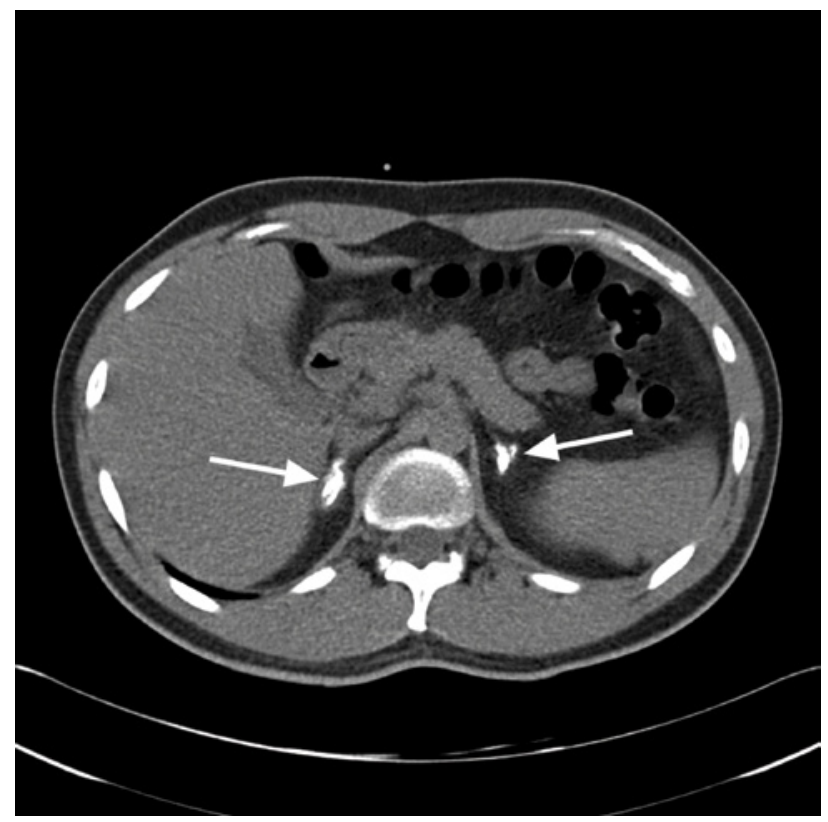

This case illustrates bilateral adrenal calcification without enlargement of the glands, possibly after an episode of adrenal haemorrhage at the age of 10 years, presumably due to an episode of septicaemia caused by Streptococcus mitis. Normal adrenal function has been maintained for 13 years.

Waterhouse-Friderichsen syndrome is classically due to meningococcal septicaemia, but may be precipitated by $S$. pneumoniae and $\beta$ haemolytic streptococcus group $A$ infection. Haemorrhage is the most common cause of adrenal calcification. In $20 \%$ of the cases it can be bilateral. Most $(90 \%)$ of the adrenal tissue must be destroyed for adrenal insufficiency. 